\title{
Riesz Basicity for General Systems of Functions
}

\author{
A. M. Sarsenbi ${ }^{1}$ and P. A. Terekhin ${ }^{2}$ \\ ${ }^{1}$ Department of Mathematics, M. Auezov South Kazakhstan State University, Tauke Han Avenue 5, Shymkent 160012, Kazakhstan \\ ${ }^{2}$ Department of Mechanics and Mathematics, N. G. Chernyshevsky Saratov State University, Astrakhanskaya 83, Saratov 410012, Russia
}

Correspondence should be addressed to A. M. Sarsenbi; abzhahan@mail.ru

Received 11 May 2014; Accepted 8 July 2014; Published 12 August 2014

Academic Editor: Józef Banaś

Copyright (c) 2014 A. M. Sarsenbi and P. A. Terekhin. This is an open access article distributed under the Creative Commons Attribution License, which permits unrestricted use, distribution, and reproduction in any medium, provided the original work is properly cited.

In this paper we find the general conditions for a complete biorthogonal conjugate system to form a Riesz basis. We show that if a complete biorthogonal conjugate system is uniformly bounded and its coefficient space is solid, then the system forms a Riesz basis. We also construct affine Riesz bases as an application to the main result.

\section{Main Result}

The aim of this paper is to find the general conditions for a complete biorthogonal conjugate system to form a Riesz basis, following the results obtained by Bari [1], Christensen [2], Sarsenbi with coauthors [3-5], San Antolin and Zalik [6], and Guo [7].

Let $\left\{u_{n}(x)\right\}_{n=1}^{\infty}$ and $\left\{v_{n}(x)\right\}_{n=1}^{\infty}$ be a complete biorthogonal conjugate system of functions from $L^{2}(0,1)$ space.

By system coefficient space $\left\{u_{n}(x)\right\}_{n=1}^{\infty}$ we denote the space $X(u)$ of all the numeric sequences $a=\left\{a_{n}\right\}_{n=1}^{\infty}$ such that the series $\sum_{n=1}^{\infty} a_{n} u_{n}(x)$ converges in $L^{2}(0,1)$. It is evident that coefficient space $X(u)$ is complete under the norm $\|a\|_{X(u)}=$ $\sup _{n \in \mathbb{N}}\left\|\sum_{k=1}^{n} a_{k} u_{k}(x)\right\|$, and a natural basis $\varepsilon_{i}=\left\{\delta_{i j}\right\}_{j=1}^{\infty}, i \in \mathbb{N}$, where $\delta_{i j}$ is a Kronecker delta, forms a $X(u)$ space basis.

A Banach coordinate space $X$ of numeric sequences $a=$ $\left\{a_{n}\right\}_{n=1}^{\infty}$ is said to be solid if $b \in X$ follows from $a \in X$ and $\left|b_{n}\right| \leq\left|a_{n}\right|, n \in \mathbb{N}$ (the inequality $\|b\|_{X} \leq\|a\|_{X}$, as it is put by the precise definition, is not required here).

It is clear that $X(u)$ space is solid if natural basis is an unconditional basis for $X(u)$. The latter follows from unconditional basicity for a system $\left\{u_{n}(x)\right\}_{n=1}^{\infty}$.

Theorem 1. Let $\left\{u_{n}(x)\right\}_{n=1}^{\infty}$ and $\left\{v_{n}(x)\right\}_{n=1}^{\infty}$ be a complete biorthogonal conjugate system of functions that is uniformly bounded:

$$
\int_{0}^{1}\left|u_{n}(x)\right|^{2} d x \leq C, \quad \int_{0}^{1}\left|v_{n}(x)\right|^{2} d x \leq C, \quad n \in \mathbb{N} .
$$

Let there be given coefficient spaces $X(u)$ and $X(v)$ which are both solid. Then $\left\{u_{n}(x)\right\}_{n=1}^{\infty}$ and $\left\{v_{n}(x)\right\}_{n=1}^{\infty}$ system form a Riesz basis.

Proof. We consider the series $\sum_{n=1}^{\infty} a_{n} u_{n}(x)$ for a numeric sequence $\left\{a_{n}\right\}_{n=1}^{\infty} \in \ell^{2}$ and show that series converges for almost all choices of signs, that is, series

$$
\sum_{n=1}^{\infty} a_{n} r_{n}(t) u_{n}(x)
$$

where $\left\{r_{n}(t)\right\}_{n=1}^{\infty}$ is the Rademacher system and converges for almost all $t \in[0,1]$ in $L^{2}$ metrics by variable $x$ (e.g., [8, Chapter 2]).

We use the results from [8] claiming that convergence of series $\sum_{n=1}^{\infty} f_{n}(x)$ for almost all choices of signs is equivalent to

$$
\left(\sum_{n=1}^{\infty}\left|f_{n}(x)\right|^{2}\right)^{1 / 2} \in L^{2}(0,1)
$$

For the series considered $\sum_{n=1}^{\infty} a_{n} u_{n}(x)$, by Levi's theorem we have

$$
\begin{aligned}
\int_{0}^{1} \sum_{n=1}^{\infty}\left|a_{n} u_{n}(x)\right|^{2} d x & =\sum_{n=1}^{\infty}\left|a_{n}\right|^{2} \int_{0}^{1}\left|u_{n}(x)\right|^{2} d x \\
& \leq C \sum_{n=1}^{\infty}\left|a_{n}\right|^{2}<\infty
\end{aligned}
$$


meaning

$$
\left(\sum_{n=1}^{\infty}\left|a_{n} u_{n}(x)\right|^{2}\right)^{1 / 2} \in L^{2}(0,1)
$$

Convergence of the series $\sum_{n=1}^{\infty} a_{n} u_{n}(x)$ for almost all choices of signs is shown.

Now take a fixed $t_{0} \in[0,1]$ such that the series $\sum_{n=1}^{\infty} a_{n} r_{n}\left(t_{0}\right) u_{n}(x)$ converges in $L^{2}(0,1)$ space. By the solidity condition for coefficient space $X(u)$ in $L^{2}(0,1)$, the series $\sum_{n=1}^{\infty} a_{n} u_{n}(x)$ converges, too.

Thus for any numeric sequence $a=\left\{a_{n}\right\}_{n=1}^{\infty} \in \ell^{2}$ the series $\sum_{n=1}^{\infty} a_{n} u_{n}(x)$ converges in $L^{2}(0,1)$. Then the following equivalent inequalities are satisfied:

$$
\begin{gathered}
\left\|\sum_{n=1}^{\infty} a_{n} u_{n}\right\|^{2} \leq B \sum_{n=1}^{\infty}\left|a_{n}\right|^{2}, \quad\left\{a_{n}\right\}_{n=1}^{\infty} \in \ell^{2}, \\
\sum_{n=1}^{\infty}\left|f, u_{n}\right|^{2} \leq B\|f\|^{2}, \quad f \in L^{2}(0,1) .
\end{gathered}
$$

This means that $\left\{u_{n}(x)\right\}_{n=1}^{\infty}$ is Bessel system.

Besselian property for a system $\left\{v_{n}(x)\right\}_{n=1}^{\infty}$ is proved in the same way. It is clear that Besselian property for both biorthogonal conjugate systems $\left\{u_{n}(x)\right\}_{n=1}^{\infty}$ and $\left\{v_{n}(x)\right\}_{n=1}^{\infty}$ implies the Riesz basicity for these systems.

Remark 2. Note that in Theorem 1 we can replace the coefficient space $X(u)$ with $X(u) \cap \ell^{2}$ and $X(v)$ with $X(v) \cap \ell^{2}$.

\section{Affine Riesz bases}

Let function $u: \mathbb{R} \rightarrow \mathbb{R}$ have a support supp $u \subset[0,1]$. Using the representation $n=2^{k}+j, k=0,1, \ldots, j=0, \ldots, 2^{k}-$ 1 for $n \in \mathbb{N}$, we assume

$$
u_{n}(x)=u_{k, j}(x)=2^{k / 2} u\left(2^{k} x-j\right) .
$$

Besides, we suppose $u_{0}(x)=1, x \in[0,1]$. System of functions $\left\{u_{n}(x)\right\}_{n=0}^{\infty}$ is called an affine system generated by a function $u$. Here and elsewhere we assume

$$
u \in L^{2}(0,1), \quad \int_{0}^{1} u(x) d x=0 .
$$

Note that the classic example of an affine system of functions is the Haar wavelet $\left\{h_{n}(x)\right\}_{n=0}^{\infty}$ generated by the function

$$
h(x)= \begin{cases}1, & x \in\left[0, \frac{1}{2}\right), \\ -1, & x \in\left[\frac{1}{2}, 1\right), \\ 0, & x \notin[0,1) .\end{cases}
$$

We enumerate the functions of Rademacher system $\left\{r_{k}\right\}_{k=0}^{\infty}$

$$
r_{k}=2^{-k / 2} \sum_{j=0}^{2^{k}-1} h_{k, j}, \quad k=0,1, \ldots
$$

We suppose that an affine system $\left\{u_{n}(x)\right\}_{n=0}^{\infty}$ generator $u$ can be represented by Rademacher system

$$
u=\sum_{k=0}^{\infty} a_{k} r_{k}, \quad \sum_{k=0}^{\infty}\left|a_{k}\right|^{2}<\infty
$$

In this case we have the following completeness criterion for a system $\left\{u_{n}(x)\right\}_{n=0}^{\infty}$. Let the function

$$
U(z)=\sum_{k=0}^{\infty} a_{k} z^{k}, \quad|z|<1,
$$

be analytic in the unit disk with coefficients $a_{k}$ from (11).

Theorem 3 (see [9]). A necessary and sufficient condition for an affine system $\left\{u_{n}(x)\right\}_{n=0}^{\infty}$ to be complete in $L^{2}(0,1)$ space is that analytic function $U(z)$ is outer function.

The following results are true for function $u$ in the form (11).

Theorem 4. System $\left\{v_{n}(x)\right\}_{n=0}^{\infty}$ that is biorthogonal conjugate to the affine system $\left\{u_{n}(x)\right\}_{n=0}^{\infty}$ exists and is complete in $L^{2}(0,1)$ space if $a_{0} \neq 0$.

Proof. Suppose

$$
V(z)=\frac{1}{U(z)}=\sum_{k=0}^{\infty} b_{k} z^{k}
$$

that is,

$$
a_{0} b_{0}=1, \quad \sum_{\nu=0}^{k} a_{\nu} b_{k-\nu}=0, \quad k \geq 1 .
$$

Then it follows from the results of [10] that

$$
v_{n}=v\left(\alpha_{1}, \ldots, \alpha_{k}\right)=\sum_{\nu=0}^{k} 2^{-(k-v) / 2} b_{k-\nu} h\left(\alpha_{1}, \ldots, \alpha_{\nu}\right)
$$

where $n \in \mathbb{N}$ and $n=2^{k}+\sum_{\nu=1}^{k} \alpha_{\nu} 2^{k-v}$ is binary expansion, $h\left(\alpha_{1}, \ldots, \alpha_{v}\right)=h_{m}$ is the Haar function for $m=2^{v}+$ $\sum_{\mu=1}^{\nu} \alpha_{\mu} 2^{\nu-\mu}$, and $v_{0}(x)=1, x \in[0,1]$. The explicit representation (15) shows that $v_{n}$ is a Haar polynomial of degree $n$. Hence it follows that the system $\left\{v_{n}(x)\right\}_{n=0}^{\infty}$ is complete.

Now we can formulate the Riesz basicity test for affine system $\left\{u_{n}(x)\right\}_{n=0}^{\infty}$ with form (11) generator, based on Theorem 1 .

Theorem 5. Let analytic function $U(z)$ have an absolutely convergent Taylor-series expansion

$$
\sum_{k=0}^{\infty}\left|a_{k}\right|<\infty
$$

and $U(z)$ does not vanish in the closed unit disk $(|z| \leq 1)$. Then an affine system of functions $\left\{u_{n}(x)\right\}_{n=0}^{\infty}$ forms a Riesz basis. 
Proof. By the conditions of the theorem, $U(z)$ is outer function. By Theorem 3, an affine system $\left\{u_{n}(x)\right\}_{n=0}^{\infty}$ is complete in $L^{2}(0,1)$ space. By Theorem 4 , biorthogonal conjugate system $\left\{v_{n}(x)\right\}_{n=0}^{\infty}$ is complete, too.

Obviously, $\left\|u_{n}\right\| \leq \max \{1,\|u\|\}$. From representation (15) we get

$$
\left\|v_{n}\right\|^{2} \leq \sum_{k=0}^{\infty} 2^{-k}\left|b_{k}\right|^{2}<\infty, \quad n \in \mathbb{N} .
$$

We need to take into account that by Wiener theorem on absolutely convergent Taylor series we have

$$
\sum_{k=0}^{\infty}\left|b_{k}\right|<\infty
$$

Finally, from results of [11] it follows that $X(u) \cap \ell^{2}=\ell^{2}$ and $X(v) \cap \ell^{2}=\ell^{2}$, so all the conditions from Theorem 1 including the Remark are satisfied.

\section{Conflict of Interests}

The authors declare that there is no conflict of interests regarding the publication of this paper.

\section{Acknowledgments}

A. M. Sarsenbi was supported by the Ministry of Education and Science of the Republic of Kazakhstan (0264/GF, 0753/GF). P. A. Terekhin was supported by the Russian Foundation for Basic Research (Grant no. 13-01-00102) and the President of the Russian Federation (Grant no. MD1354.2013.1). The results of Section 2 were obtained within the framework of the state task of Russian Ministry of Education and Science (Project 1.1520.2014K).

\section{References}

[1] N. K. Bari, "Biorthogonal systems and bases in Hilbert space," Uchenye Zapiski Moskovskogo Gosudarstvennogo Universiteta, vol. 4, no. 148, pp. 69-107, 1951.

[2] O. Christensen, An Introduction to Frames and Riesz Bases, Birkhäuser, Boston, Mass, USA, 2003.

[3] A. Kopzhassarova and A. Sarsenbi, "Basis properties of eigenfunctions of second-order differential operators with involution," Abstract and Applied Analysis, vol. 2012, Article ID 576843, 6 pages, 2012.

[4] A. A. Kopzhassarova, A. L. Lukashov, and A. M. Sarsenbi, "Spectral properties of non-self-adjoint perturbations for a spectral problem with involution," Abstract and Applied Analysis, vol. 2012, Article ID 590781, 5 pages, 2012.

[5] A. M. Sarsenbi, "Necessary and sufficient conditions for the Riesz basis property of the eigen- and associated functions of high-order differential operators on an interval," Doklady Mathematics, vol. 77, no. 2, pp. 290-292, 2008.

[6] A. San Antolin and R. A. Zalik, "Some bivariate smooth compactly supported tight framelets with three generators," Abstract and Applied Analysis, vol. 2013, Article ID 818907, 7 pages, 2013.
[7] X. Guo, “g-bases in Hilbert spaces," Abstract and Applied Analysis, vol. 2012, Article ID 923729, 14 pages, 2012.

[8] B. S. Kashin and A. A. Sahakian, Orthogonal Series, AMS, Providence, RI, USA, 1989.

[9] P. A. Terekhin, "A multishift on a Hilbert space," Functional Analysis and Its Applications, vol. 39, no. 1, pp. 57-67, 2005.

[10] P. A. Terekhin, "Convergence of biorthogonal series in the system of contractions and translations of functions in the spaces $L^{P}[0,1]$," Mathematical Notes, vol. 83 , no. 5-6, pp. 657674, 2008.

[11] P. A. Terekhin, "Riesz bases generated by contractions and translations of a function on an interval," Mathematical Notes, vol. 72, no. 3-4, pp. 547-560, 2002. 


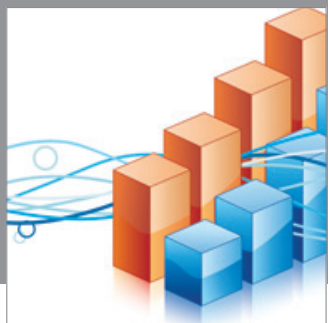

Advances in

Operations Research

mansans

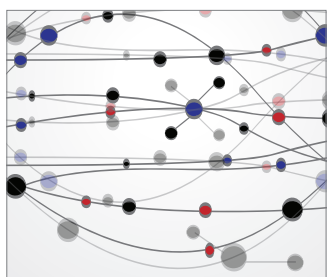

The Scientific World Journal
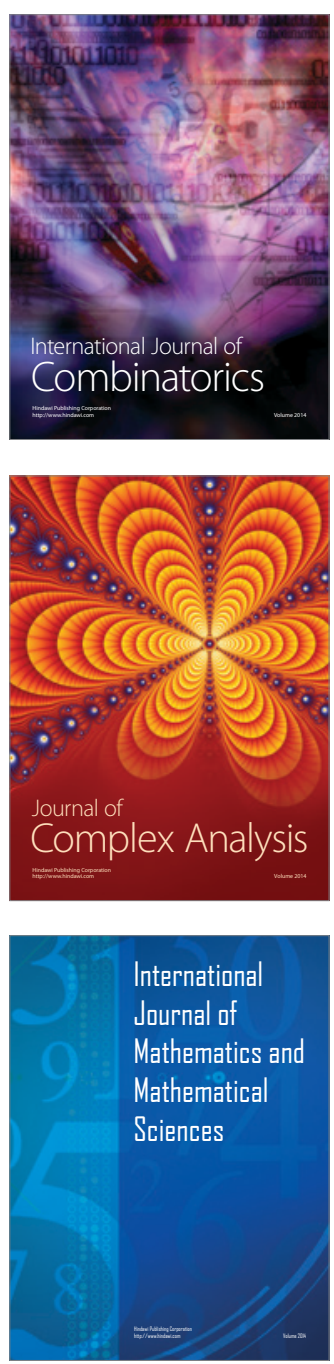
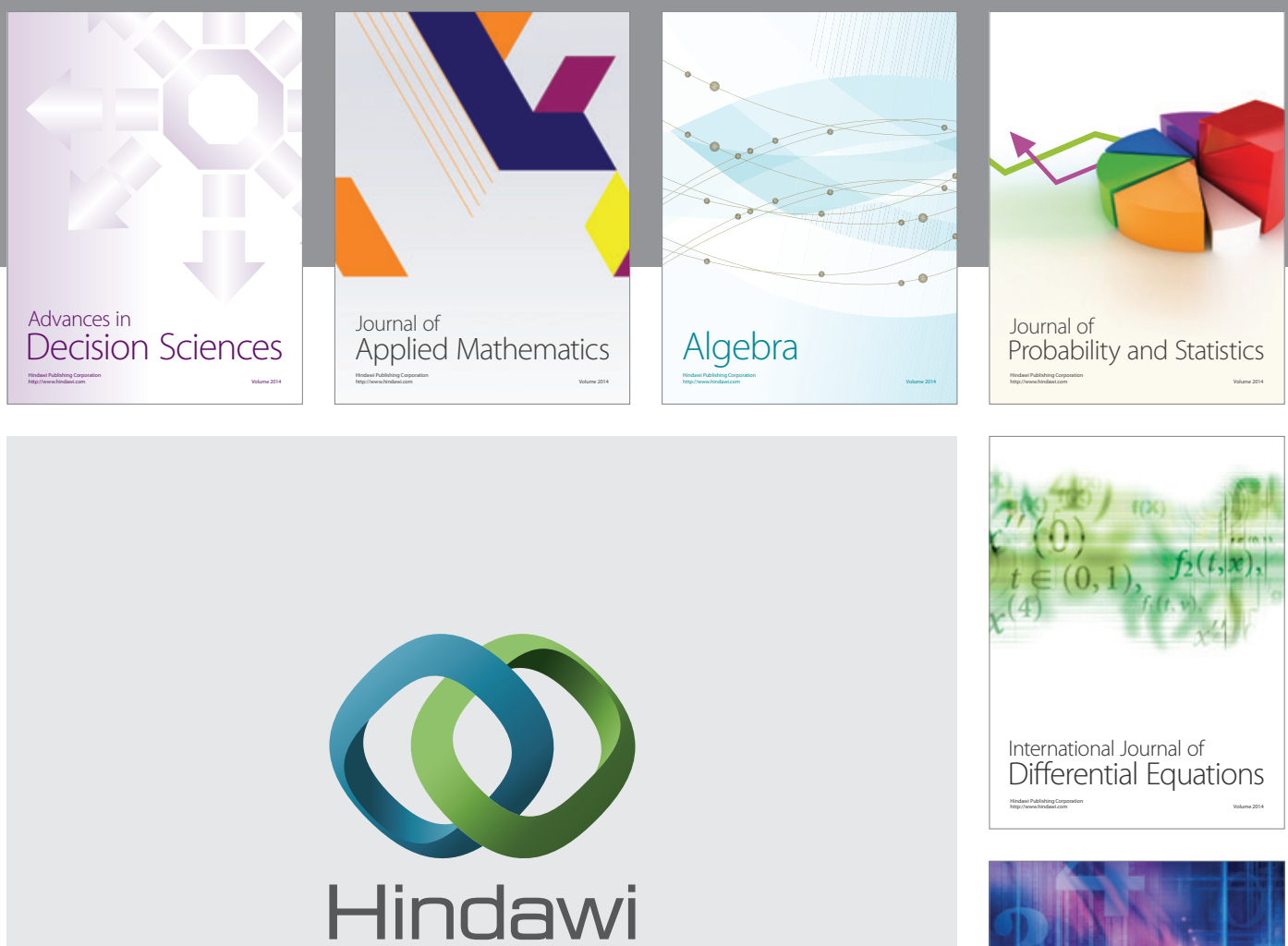

Submit your manuscripts at http://www.hindawi.com
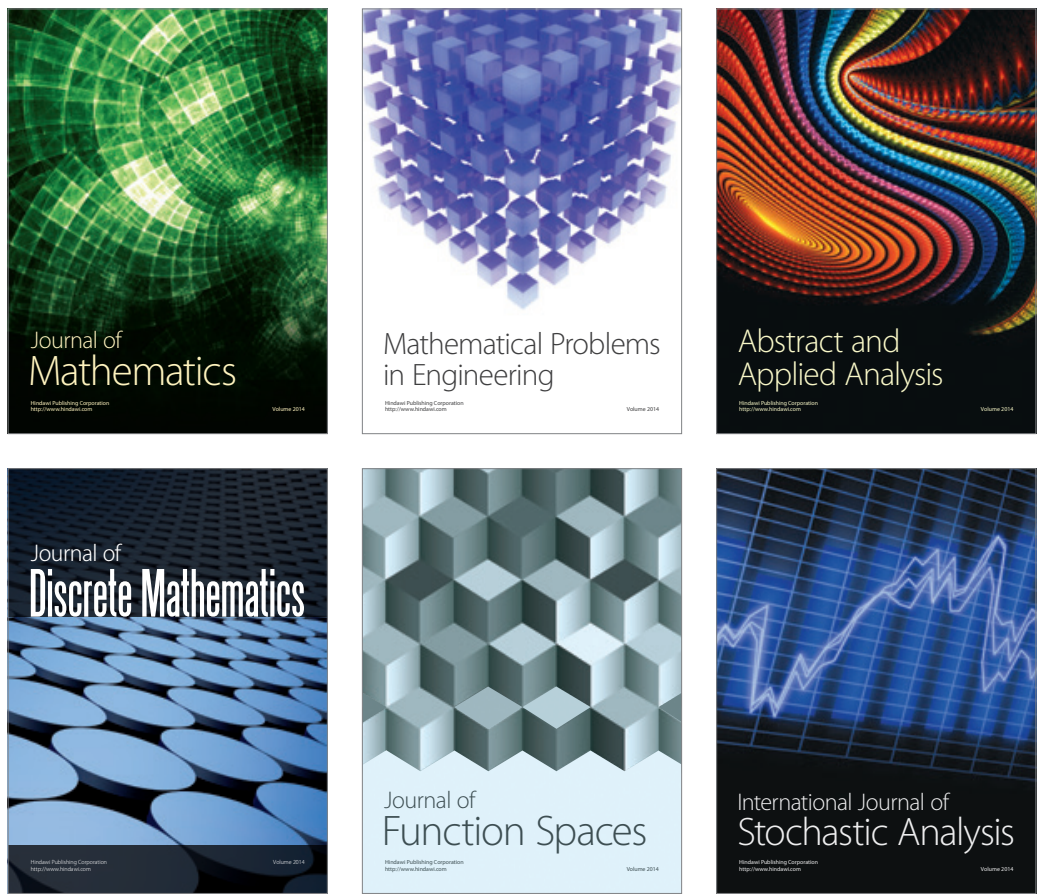

Journal of

Function Spaces

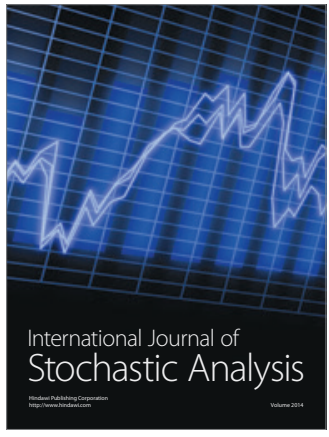

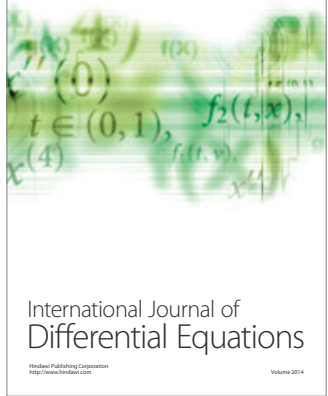
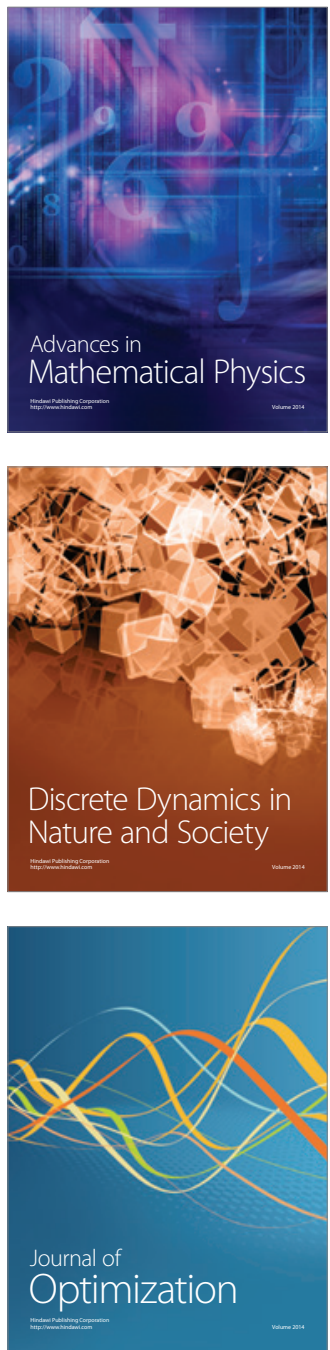